\title{
Transplantation of adipose-derived stem cells for peripheral nerve repair
}

\author{
GUIBO LIU ${ }^{1,2}$, YONGXIA CHENG ${ }^{3}$, SUFEN GUO $^{3}$, YUKUAN FENG $^{2}$, QI LI $^{1}$, \\ HUA JIA $^{1}$, YING WANG ${ }^{1,2}$, LEI TONG $^{1}$ and XIAOJIE TONG ${ }^{1}$ \\ ${ }^{1}$ Department of Anatomy, College of Basic Medical Sciences, China Medical University, Shenyang 110001; \\ ${ }^{2}$ Departments of Anatomy and ${ }^{3}$ Pathology, Mudanjiang Medical College, Mudanjiang 157011, P.R. China
}

Received April 5, 2011; Accepted May 19, 2011

DOI: $10.3892 / \mathrm{ijmm} .2011 .725$

\begin{abstract}
Mesenchymal stem cells have become a very attractive source of cell implantation for neural tissue engineering. The ideal stem cells for transplantation should be easily obtained, and should rapidly proliferate in vitro and have low immunogenicity. The purpose of this study was to investigate the regenerative potential of adipose-derived stem cells (ADSC) on peripheral nerve repair. ADSCs were isolated from rat adipose tissue and cultured until adherent cells became morphologically homogeneous with a fibroblast-like shape, and transplanted with acellular nerve allografts (ANAs) into rat models with a $10 \mathrm{~mm}$ gap of transected sciatic nerve defect. After cell transplantation, we found that ADSC implantation improved functional recovery of exercise behavior and increased wet weight ratio of the anterior tibial muscle. In the electrophysiological testing, we found that the percentage of activated fibers was higher in the ADSC-implanted animals as evidenced by the increase of nerve conduction velocity and amplitude. Histological examination revealed that the number of nerve fibers, axonal diameter and myelin thickness were significantly higher in the ADSC-implanted animals compared to the control. In addition, we demonstrated that the progression of the regenerative process after ADSC implantation was accompanied by elevated expression of neurotrophic factors at both the early and later phase. Taken together, these results suggest that ADSCs can promote the repair of peripheral nerve injury, and the combination of ADSC and ANA transplantation is a new therapeutic method for long distant peripheral nerve defects. Our data also provide evidence indicating the strong association of neurotrophic factor production to the regenerative potential of implanted ADSCs.
\end{abstract}

Correspondence to: Dr Xiaojie Tong, Department of Anatomy, College of Basic Medical Sciences, China Medical University, 92 North Second Road, Shenyang 110001, P.R. China

E-mail: xiaojie_tong@163.com

Key words: adipose-derived stem cell, transplantation, peripheral nerve repair, acellular nerve allograft, neurotrophic factors

\section{Introduction}

Traditional therapeutic approaches for the reconstruction of peripheral nerve defects include end-to-end suturing, fascicular suturing, nerve graft, and nerve conduits. There is evidence indicating that nerve grafting is essential for reconstruction of long nerve defects. Recently, neural tissue engineering has received much attention, and Schwann cell transplantation has been reported to achieve reliable outcomes in the regeneration of the sciatic nerve (1-3). However, Schwann cell isolation can cause additional damage, and these cells need a long time for cell culture and growth, which have limited their clinical application.

Mesenchymal stem cells (MSC) have recently become a very attractive source of cell implantation for tissue engineering because of their self-proliferation, fast proliferation and multilineage differentiation potential (4). A large number of studies have shown that bone marrow-derived mesenchymal stem cells (BMMSC) can promote the repair of peripheral nervous system injury (5-11). Chen et al (11) found that rat BMMSC can synthesize and secrete a number of neurotrophic factors (NF), including nerve growth factor (NGF), brain-derived neurotrophic factor (BDNF), ciliary neurotrophic factors (CNTF) and glial cell line-derived neurotrophic factor (GDNF). They showed the strong association of NF production with the regenerative potential of implanted BMMSC in the process of peripheral nerve repair, thus providing the mechanism underlying the regenerative potential of BMMSC on peripheral nerve repair. However, BMMSC collection and preparation is a highly invading process and the number of mesenchymal stem cells in the bone marrow is relatively low (12). Therefore, it is urgent to find alternative sources of stem cell for transplantation.

Since Zuk et al (12) reported on adipose-derived stem cells (ADSC), mesenchymal stem cells derived from fat tissue, they have become the focus of research in regenerative medicine because of their unique characteristics. ADSCs have similar phenotype and gene expression profiles to BMMSCs (13-15). However, ADSCs have some unique advantages, such as that they are more easily obtained from rich fat sources, they have a higher ratio in adipose tissue, low immunogenicity, and a faster proliferation rate than BMMSCs (16-18).

An autologous nerve graft can provide structural support sprouting axons originating from the proximal nerve stump 
and has been traditionally used in the treatment of peripheral nerve defects (19). It has been proven that the internal structure of nerve grafts and extracellular matrix components play a key role in cell migration and axon extending $(20,21)$. However, the major shortcomings of the autologous nerve graft include multiple surgeries, the requirement to sacrifice a healthy nerve, and shortage of graft material. Therefore, an alterative to the autograft is needed. Sondell et al (22) have demonstrated that extraction with the detergents Triton X-100 and deoxycholate results in acellular nerve allografts (ANA), an endogenous nerve segments with preserved basal lamina tubes. These acellular nerves have similar structures with autologous nerve distribution and biochemical characteristics and can be transplanted into the body as a natural scaffold, to which surrounding cells readily migrate, thus forming the foundation for nerve regeneration.

In this study, we aimed to investigate the mechanism and regenerative potential of ADSC implantation on peripheral nerve injury. We found that ADSCs can promote the repair of peripheral nerve injury, which may be related to the synthesis and secretion of neurotrophic factors.

\section{Materials and methods}

Animals. Wistar rats (3-4 weeks old), weighing 200-250 g, obtained from the Experimental Animal Centre of China Medical University, were used in this study. The animal study protocol was reviewed and approved by the Animal Experimental Committee of the China Medical University. If not stated otherwise, anesthetization of animals was achieved using $10 \%$ chloral hydrate $(350 \mathrm{~g} / \mathrm{kg})$ in this study.

ADSC isolation and culture. ADSCs were isolated from adult Wistar rat adipose tissue as previously described (23). Briefly, adipose tissue from Wistar rat groin fat pads was washed in PBS and fully cut into pieces and digested with $0.1 \%$ collagenase I at $37^{\circ} \mathrm{C}$ for $60 \mathrm{~min}$. The dissociated cells were cultured in Dulbecco's modified Eagle's medium (DMEM) containing $10 \%$ fetal bovine serum (FBS) for $48 \mathrm{~h}$. The non-adherent cells were removed. The adherent cells were continuously cultured and then used for the experiment.

Differentiation studies. ADSCs (passage 3) were cultured to confluence and changed to osteogenic medium (DMEM supplemented with $10 \% \mathrm{FBS}, 0.1 \mu \mathrm{M}$ dexamethasone, $10 \mu \mathrm{M}$ $\beta$-glycerol phosphate and $50 \mu \mathrm{M}$ ascorbate) and adipogenic medium (DMEM supplemented with 10\% FBS, $1 \mu \mathrm{M}$ dexamethasone, $5 \mu \mathrm{g} / \mathrm{ml}$ insulin, $0.5 \mathrm{mM}$ isobutylmethylxanthine and $60 \mu \mathrm{M}$ indomethacin) for 7-14 days. The differentiation potential for osteogenesis was assessed by the mineralization of calcium deposits by von Kossa histochemical staining. The adipogenesis was evaluated by production of intracellular lip droplets detected by Oil Red O staining.

In vivo ADSCs tracking. ADSCs implanted in vivo were prelabeled with the PKH26 Red Fluorescent Cell Linker (Sigma-Aldrich, St. Louis, MO, USA). ADSCs were treated with trypsin and EDTA and the cell suspension was incubated with PKH for 2-5 min. After blocking the residual dye with calf serum, three additional washings with PBS, and incubation in
1\% BSA for 1 min, the PKH26-marked cell suspension was then implanted into the experimental rat. Fluorescent Cell Linker kits provide fluorescence labeling of live cells over an extended period of time, with no apparent toxic effects. Labeled cells retain both biological and proliferative activity, and are ideal for cell tracking studies. The PKH-26-labeled cells were analyzed under a fluorescence microscope.

Preparation of acellular nerve allograft (ANA). The ANA was prepared as previously described with the following modification (24). Briefly, Wistar rats were anesthetized before surgery. Bilateral sciatic nerve segments ( $15 \mathrm{~mm}$ in length) were excised under sterile conditions. After removal of fat and connective tissue, the nerve segments were agitated in deionized distilled water $\left(\mathrm{ddH}_{2} \mathrm{O}\right)$ for $24 \mathrm{~h}$, and treated with $0.05 \mathrm{M}$ Tris- $\mathrm{HCl}$ and protease inhibitors in $\mathrm{ddH}_{2} \mathrm{O}$ for 4 days, followed by treatment with $4 \%$ Triton X-100 (Sigma-Aldrich) in $\mathrm{ddH}_{2} \mathrm{O}$ for an additional $24 \mathrm{~h}$. After thorough washing in PBS, the treated nerves were stored in $10 \mathrm{mM}$ PBS at $-80^{\circ} \mathrm{C}$ until use.

Surgical procedures. After the animals were anesthetized, the right sciatic nerve was exposed and removed $(8 \mathrm{~mm})$ near the obturator tendon in mid-thigh. A $10 \mathrm{~mm}$-long gap was created after the nerve retraction. With regard to reconstruction of the nerve defects, the animals were randomly allocated to three groups ( $\mathrm{n}=16$ for each group), to receive implantation of autografts, ANA injected with ADSC, and ANA injected with DMEM medium. For autografting, the $1 \mathrm{~cm}$ transected sciatic nerve segment was reversely connected to the stumps (autograft group). The $10 \mathrm{~mm}$-long ANA injected with ADSC was connected to the sciatic nerve and filled the gap (ADSC group). The $10 \mathrm{~mm}$-long ANA injected with an equal volume of the DMEM culture medium connected to the sciatic gap were used as mock control (DMEM group). The wound was subsequently closed in layers. Six animals in each group were sacrificed 3 days after surgery and the rest of the animals were sacrificed 12 weeks after surgery.

Functional assessment. Functional evaluation of sciatic nerve regeneration was expressed by the sciatic function index (SFI) as previously described (11). Briefly, 12 weeks after surgery, the rats' hind feet were dipped in ink and the rats were allowed to walk across a plastic tunnel so that the footprints could be recorded on paper loaded onto the bottom of the tunnel. The distance between the third toe and heel (PL), first and fifth toe (TS), and second and fourth toe (ITS) was measured on the experimental side (EPL, ETS and EITS, respectively) and the contralateral normal side (NPL, NTS and NITS, respectively). The SFI was calculated as follows: SFI $=-38.3(\mathrm{EPL}-\mathrm{NPL}) /$ NPL + 109.5 (ETS - NTS)/NTS + 13.3 (EITS - NITS)/NITS -8.8. In general, the SFI oscillates around 0 for normal nerve function, whereas around $-100 \mathrm{SFI}$ represents total dysfunction.

Electrophysiological study. At 12 weeks post-surgery, the rats were anesthetized. The sciatic nerves were re-exposed and dissected free from surrounding tissues. Electrophysiological tests were performed using an electroneurography instrument (Haishen Medical Electronic Instrument Co., Ltd., Shanghai, China). Electric stimulation (duration of $0.1-0.2 \mathrm{msec}$, frequency of $1 \mathrm{~Hz}$, and intensity of $1-20 \mathrm{mM}$ ) was applied to 
Table I. Primer sequences used for RT-PCR.

\begin{tabular}{lllc}
\hline Gene symbol & $\begin{array}{c}\text { GenBank } \\
\text { accession no. }\end{array}$ & \multicolumn{1}{c}{ Sequence } & $\begin{array}{c}\text { Product size } \\
(\text { bp })\end{array}$ \\
\hline BDNF & NM_012513 & $\begin{array}{l}\text { Forward: 5'-GGTCACAGTCCTGGAGAAAG-3' } \\
\text { Reverse: 5'-GTCTATCCTTATGAACCGCC-3' }\end{array}$ & 214 \\
NGF & NM_227525 & $\begin{array}{l}\text { Forward: 5'-GCCCACTGGACTAAACTTCAGC-3' } \\
\text { Reverse: 5'-CCGTGGCTGTGGTCTTATCTC-3' }\end{array}$ & 349 \\
NT-3 & NM_031073 & $\begin{array}{l}\text { Forward: 5'-TGCAGAGCATAAGAGTCACC-3' } \\
\text { Reverse: 5'-AAGTCAGTGCTCGGACGTAG-3' }\end{array}$ & 269 \\
CNTF & NM_013166 & $\begin{array}{l}\text { Forward: 5'-AGAACCTCCAGGCTTACC-3' } \\
\text { Reverse: 5'-AATGGCTACATCTGCTTATC-3' }\end{array}$ & 385 \\
GDNF & NM_019139 & $\begin{array}{l}\text { Forward: 5'-GGATGTCGTGGCTGTCTG-3' } \\
\text { Reverse: 5'-GCCGCTTGTTTATCTGGT-3' }\end{array}$ & 240 \\
LIF & NM_022196 & $\begin{array}{l}\text { Forward: 5'-CCGTGTCACGGCAACCTCA-3' } \\
\text { Reverse: 5'-GGGGACACAGGGCACATCC-3' }\end{array}$ & 395 \\
\hline
\end{tabular}

the proximal side of the injured sites, and a recording electrode was placed in the extensor digitorum. The distance between two electrodes was measured by a precision of direct measurement of $0.2 \mathrm{~mm}$ vernier caliper. The onset latency, peak amplitude and the nerve conduction velocity were recorded.

Muscle weight measurement. Following electrophysiological and functional analysis, the animals were sacrificed and the anterior tibial muscles were collected. The muscles were weighed and the muscle wet weight ratio was determined by the following equation: wet weight ratio = experiment site muscle wet weight/contralateral normal site muscle wet weight $\mathrm{x} 100 \%$.

Histological examination. The middle region of the nerve grafts were fixed with $2.5 \%$ glutaraldehyde solution and placed in $4 \%$ osmium tetroxide for $2 \mathrm{~h}$. The nerve tissues were then serially dehydrated with ethanol, and embedded in paraffin. Cross-sections $(4 \mu \mathrm{m})$ were cut and stained with toluidine blue for examination by light microscopy. In addition, ultrathin sections $(0.5 \mu \mathrm{m})$ were cut and stained with uranyl acetate and lead citrate. The ultrastructural examination was carried out with a transmission electron microscope (JEM-1200EX, JEOL Electronics Co., Ltd., Tokyo, Japan). Images of the histological sections were digitized and subsequently analyzed using an image analysis system (MetaMorph/DP10/BX-41, UIC/Olympus, US/JP). For the nerve evaluation, the number of nerve fibers, the nerve fiber diameter, and the myelin sheath thickness were examined.

Tissue preparation and immunofluorescence staining. At 12 weeks post-surgery, 4 rats from each group were randomly selected and sacrificed. The nerve grafts were fixed with $4 \%$ paraformaldehyde overnight, and washed in PBS 3 times. After being placed in $30 \%$ sucrose solution overnight, the nerve tissues were embedded in optimum cutting temperature (OCT, Sigma-Aldrich). Frozen sections $(8 \mu \mathrm{m})$ were cut and stained with S-100 primary antibody (1:150, DAKO,
Denmark) overnight at $4^{\circ} \mathrm{C}$, then washed with PBS and incubated with a secondary FITC-conjugated goat anti-rabbit IgG antibody (1:200, Santa Cruz Biotechnology, Santa Cruz, CA, USA) at room temperature for $40 \mathrm{~min}$. The slides were then coverslipped and observed under a fluorescence microscope.

RNA isolation and RT-PCR. Frozen nerve grafts tissue were homogenized and total-RNA was isolated using cold TRIzol reagent (Invitrogen, Carlsbad, CA) following the manufacturer's instructions. Total-RNA was quantified by spectrophotometry and $1 \mu \mathrm{g}$ of total-RNA was used to make cDNA in a total of $20 \mu \mathrm{l}$ using the Superscript first strand synthesis kit system according to the manufacturer's protocol (Takara, Dalian, China). The PCR reaction was carried out in $45 \mu \mathrm{l}$ of reaction mixture containing $10 \mu \mathrm{l}$ of PCR buffer, $0.5 \mu \mathrm{M}$ of forward or reverse primers, and $2.0 \mu \mathrm{l}$ template cDNA on a PCR system (Biometra, Göttingen, Germany). The primer sequences, conditions, and product sizes of PCR are summarized in Table I. The relative target gene expression level was normalized on the basis of $\beta$-actin expression as an endogenous RNA control. All experiments were performed in triplicate and repeated three times.

Statistical analysis. The experiments for each group were run in triplicate. Data are expressed as the means \pm standard deviation (SD). Statistical analysis was performed using the SPSS13.0 software (SPSS, Inc., Chicago, IL, USA). A P-value $<0.05$ was considered statistically significant.

\section{Results}

Characterization of cultured ADSCs. ADSCc obtained from the inguinal fat pad of Wistar rats showed adherence and expansion after seeding and growing in culture plates for $24 \mathrm{~h}$. The adherent cells exhibited short spindle, polygonal, and large flattened appearance. After a second passage, most cells grew exhibiting a fibroblast-like morphology and reached confluence (Fig. 1A). At passage 4 the fibroblast-like 

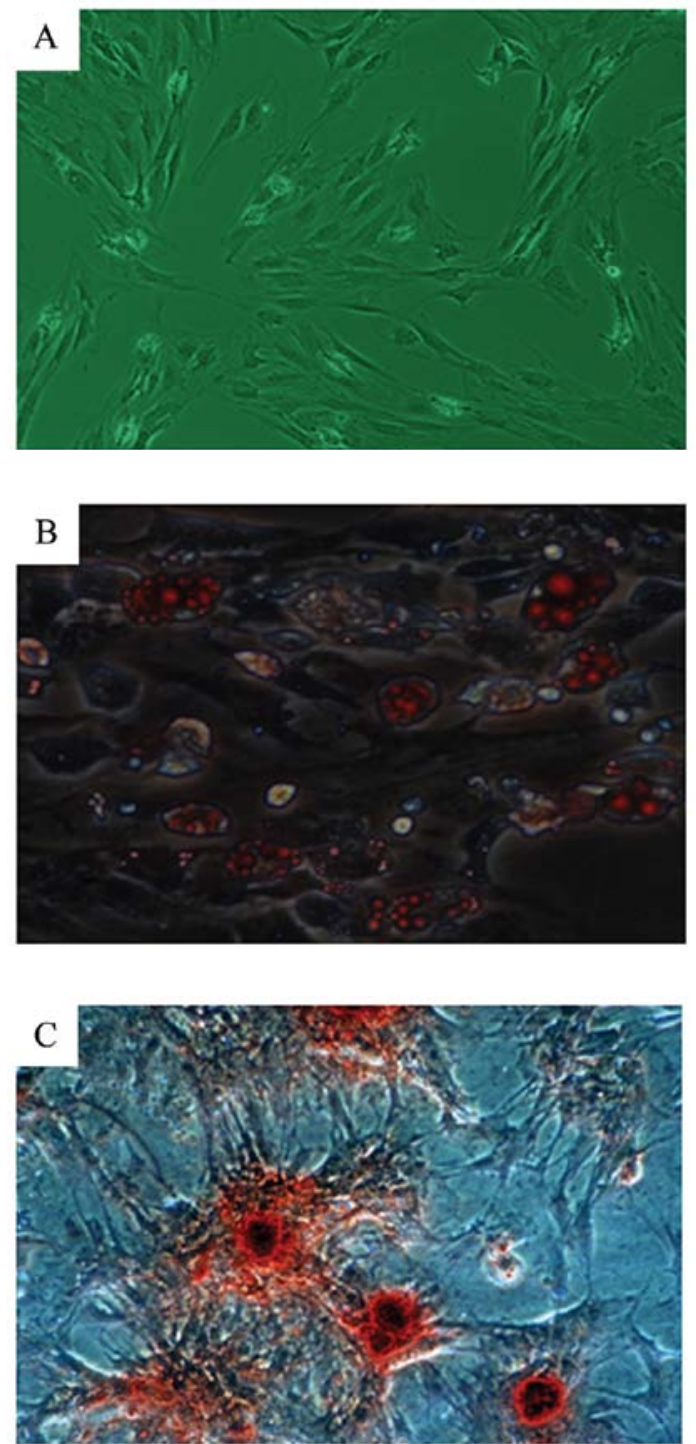

Figure 1. Characterization of cultured ADSCs. (A) Primary cultured ADSCs exhibited spindle, polygonal, cobblestone-like morphology in the first passage of the initial culture. (B) ADSCs (passage 3) were subjected to adipogenic differentiation induction for 9 days. After differentiation, the accumulation of intracellular lipid droplets was detected by Oil Red O staining. (C) After culturing ADSCs in osteogenic medium for 4 weeks, the deposition of calcium was determined by von Kossa histochemical staining. Magnification, x200.

cells became morphologically homogeneous exhibiting dense growth with aggregate-like cell clusters. After culturing the fibroblast-like cells (at passage 3) in adipogenic medium, the accumulation of intracellular lipid droplets was detected by Oil Red O staining (Fig. 1B), suggesting the adipogenic differentiation potential. When culturing the fibroblast-like cells in osteogenic medium, the differentiation potential for osteogenesis was demonstrated by the mineralization of calcium deposits by von Kossa histochemical staining (Fig. 1C). These findings reveal that the fibroblast-like cells possess differentiation potential and demonstrate their stem cell characteristics.

Functional recovery of the injured peripheral nerve. To evaluate the efficacy of the ADSCs implantation on the functional recovery in the sciatic nerve injured rats, we performed the walking track analysis and compared the SFI between
A

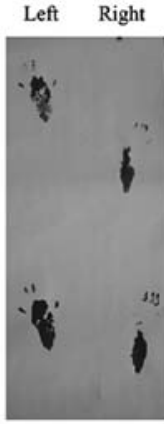

DMEM

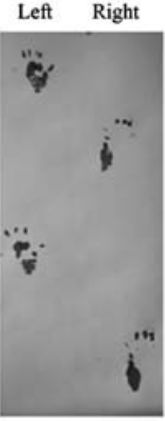

ADSC
Left Right

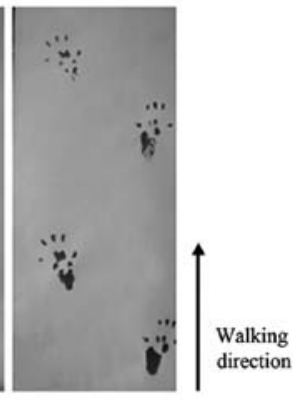

Autograft
B

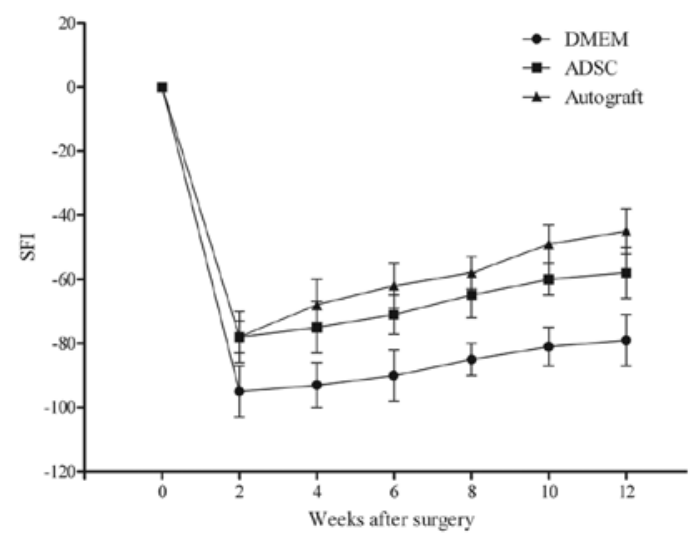

Figure 2. Walking track analysis. (A) Typical walking tracks were obtained from the autograft, ADSC-implanted, and DMEM groups 6 weeks after surgery. (B) The functional parameters were calculated in each group $(n=10$ for each group) after surgery as described in Materials and methods. The sciatic function index of the ADSC-implanted group was significantly improved compared to the DMEM group, but was similar to the autograft group. ${ }^{*} \mathrm{P}<0.05$

the ADSC-implanted, DMEM, and autograft groups. As shown in Fig. 2, typical working tracks obtained from each group at 6 weeks after surgery were depicted (Fig. 2A). The SFI of the ADSC group was significantly improved compared to the DMEM group, but there was no obvious difference in comparison with the autograft group (Fig. 2B), suggesting that ADSC implantation improved functional recovery similarly to the autograft group. Electrophysiological analysis showed that the impaired nerve conduction latency, conduction velocity, and peak amplitude after nerve injury was improved in the ADSCimplanted rats compared with the DMEM control group, but there was no difference compared to the autograft group (Fig. 3 and Table II). After the sciatic nerve injury, the target muscle degenerated and lost weight. We therefore compared the recovery of the anterior tibial muscle. The weight of the anterior tibial muscle was significantly recovered in the ADSC and autograft groups but it failed to recover in the DMEM group (Fig. 4). There was no obvious difference between the ADSC and autograft groups. These data suggest that ADSC implantation improved functional recovery after the sciatic nerve injury.

Histomorphological analysis of nerve tissues. In accordance with the functional results, histological examination showed 
Table II. The quantitative analysis of nerve conduction velocity, onset latency and peak amplitude at 12 weeks after surgery in each group $(\mathrm{n}=10)$.

\begin{tabular}{lccc}
\hline Group & Velocity $(\mathrm{m} / \mathrm{sec})$ & Latency $(\mathrm{msec})$ & Amplitude $(\mathrm{mV})$ \\
\hline DMEM & $8.19 \pm 0.89$ & $2.85 \pm 0.21$ & $13.11 \pm 1.41$ \\
ADSC & $12.45 \pm 0.76^{\mathrm{a}}$ & $1.38 \pm 0.14^{\mathrm{a}}$ & $27.12 \pm 1.48^{\mathrm{a}}$ \\
Autograft & $14.24 \pm 1.16$ & $1.04 \pm 0.14$ & $29.50 \pm 1.61$ \\
\hline
\end{tabular}

${ }^{\text {aP }}<0.05$ vs. the DMEM group.

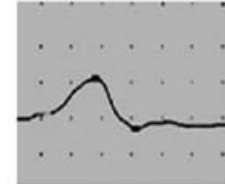

DMEM

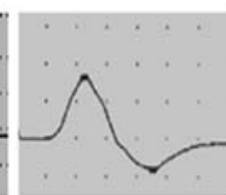

ADSC

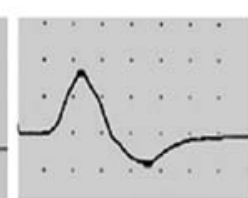

Autograft
Figure 3. Electrophysiological analysis. The representative electromyographic recording waves were elicited in response to electrical stimulation of the sciatic nerve proximal to the lesion in the autograft, ADSC-implanted, and DMEM groups 12 weeks after surgery.

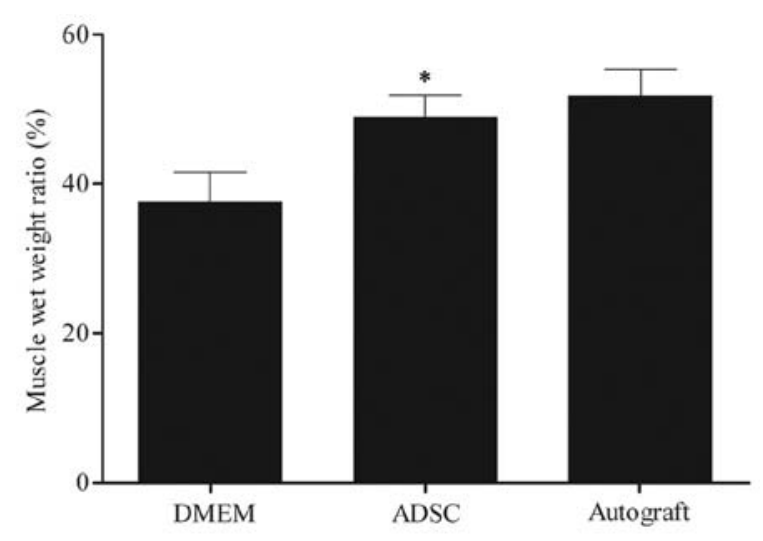

Figure 4. Measurement of the anterior tibial muscle. The anterior tibial muscles of both sides were removed from the autograft, ADSC-implanted, and DMEM groups ( $\mathrm{n}=10$ for each group) and weighed 12 weeks after surgery. The wet weight ratios were calculated as described in Materials and methods. ${ }^{*} \mathrm{P}<0.05$.

obvious regeneration of the nerve tissue in the ADSC group. Toluidine blue staining revealed that the number of nerve fibers, axonal diameter and myelin thickness were significantly higher in the ADSC group compared to that in the DMEM group, but similar to that in the autograft group (Fig. 5 and Table III). Electron microscopic examination showed that the nerve fibers from the DMEM group displayed uneven axonal diameter and obvious degeneration. However, in the ADSC and autograft groups, the nerve fibers showed slight degeneration with uniform axonal diameters (Fig. 6). The findings suggest that ADSC implantation promoted the regeneration of the sciatic nerve as evidenced by the axonal outgrowth and remyelination.
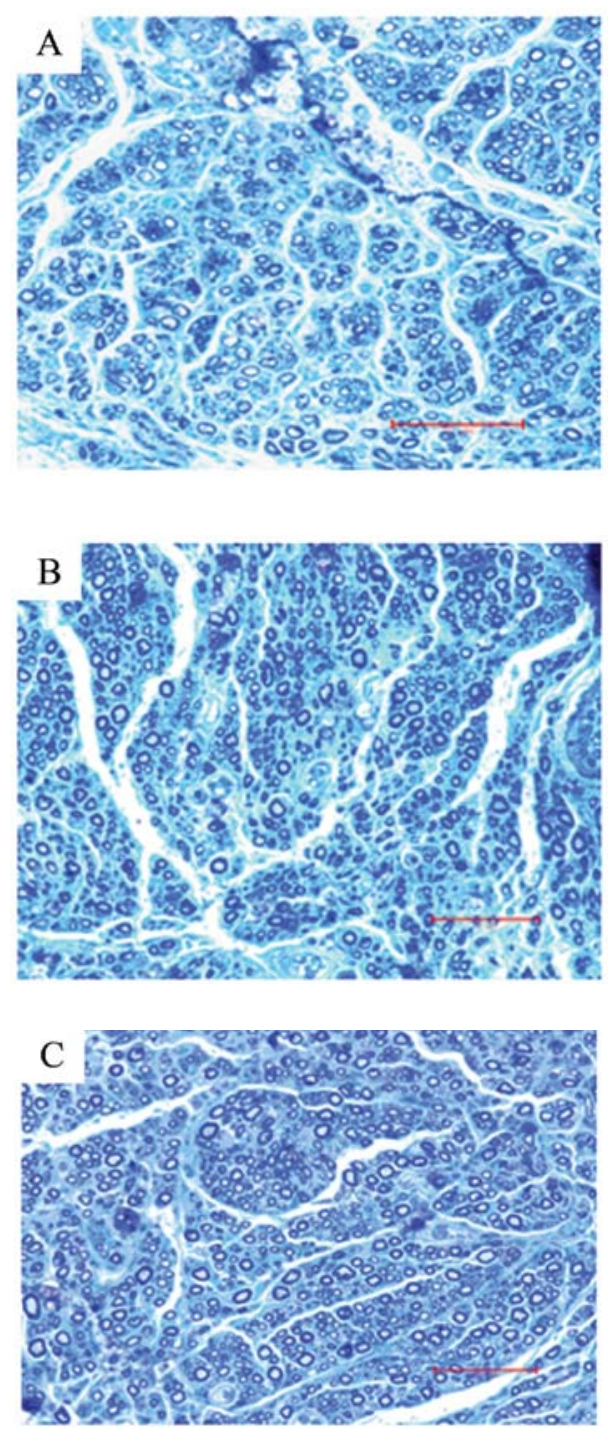

Figure 5. Histological examination of nerve tissue. Cross sections of nerve grafts from the (A) DMEM, (B) ADSC-implanted and (C) autograft groups at 12 weeks after surgery were stained with toluidine blue. Scale bar, $50 \mu \mathrm{m}$. $\mathrm{n}=10$.

Observation of ADSCs in nerve grafts. In order to confirm that the ADSCs were successfully implanted in peripheral nerve injured rats, we tracked the PKH26-labeled ADSCs in the nerve graft. At 12 weeks after surgery, the nerve grafts were found to be connected to both stumps of the sciatic nerve (Fig. 7A). Using immunofluorescence microscopy, PHK26- 
Table III. The quantitative analysis of axon number, axon diameter and myelin thickness at 12 weeks after surgery in each group $(\mathrm{n}=10)$.

\begin{tabular}{lccc}
\hline Group & $\begin{array}{c}\text { Axon number } \\
\left(\mathrm{n} / 2,500 \mathrm{~mm}^{2}\right)\end{array}$ & Axon diameter $(\mu \mathrm{m})$ & Myelin thickness $(\mu \mathrm{m})$ \\
\hline DMEM & $32.12 \pm 0.82$ & $0.47 \pm 0.08$ & $2.01 \pm 0.12$ \\
ADSC & $53.45 \pm 1.22^{\mathrm{a}}$ & $0.81 \pm 0.12^{\mathrm{a}}$ & $4.26 \pm 0.27^{\mathrm{a}}$ \\
Autograft & $61.41 \pm 1.57$ & $1.03 \pm 0.15$ & $5.13 \pm 0.52$ \\
\hline
\end{tabular}

${ }^{\mathrm{a} P}<0.05$ vs. DMEM group.
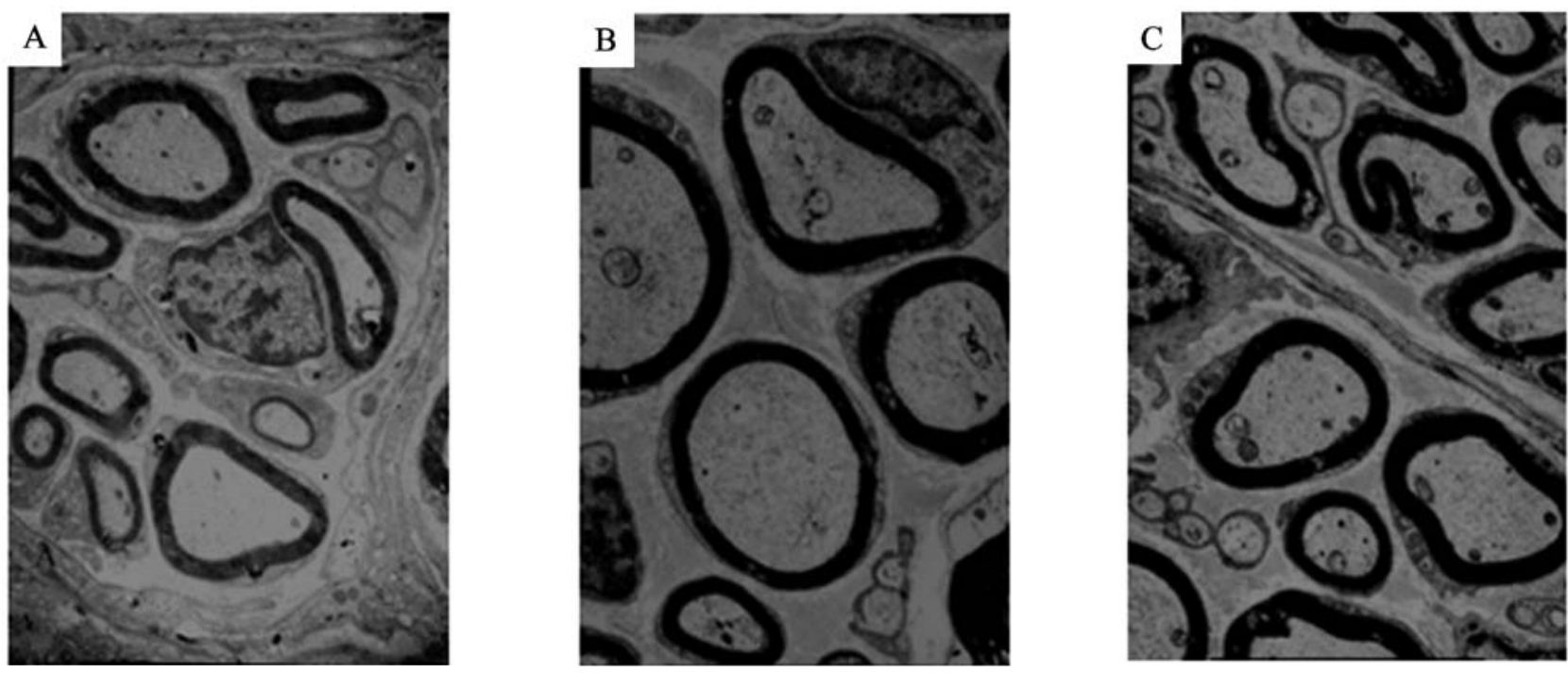

Figure 6. Electron microscopic examination of regenerative nerve tissue. Scanning electron micrographs of cross sections of the nerve tissue demonstrate that the nerve fibers from the (A) DMEM group animals displayed uneven axonal diameter. By contrast, the animals from the (B) ADSC-implanted and (C) autograft groups showed uniform axonal diameter of the regenerative nerve tissue. Magnification, x6,000.
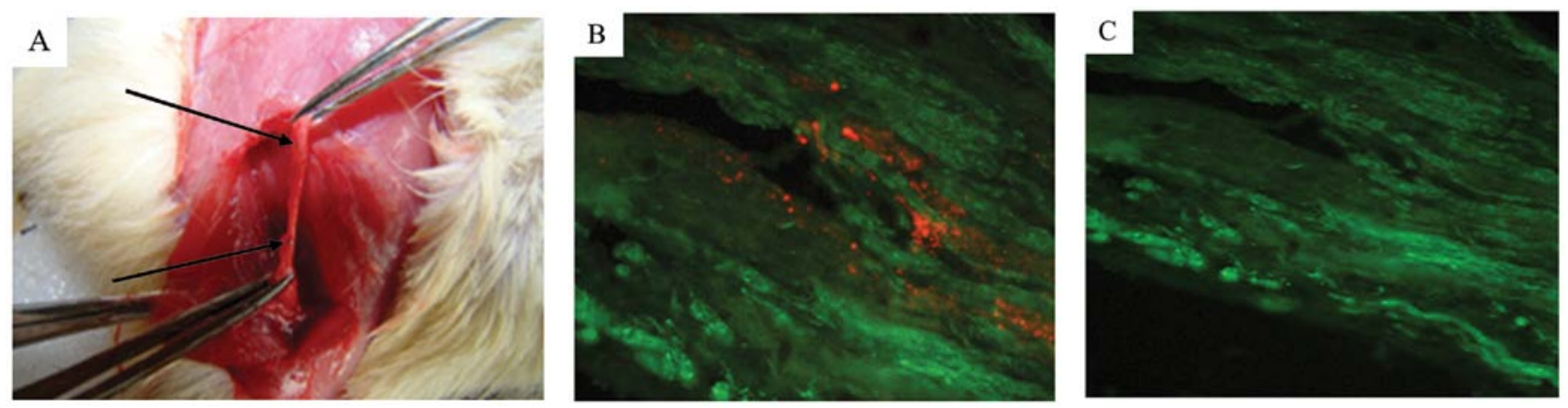

Figure 7. Immunofluorescence staining of ADSCs in nerve grafts. (A) Intraoperative photograph showing that the nerve grafts were connected to both stumps of the sciatic nerve 12 weeks after surgery. The arrows show the suture connection sites of the nerve graft. Microscopic immunodetection of PHK26 (red) and S-100 (green) showed PHK26-labeled ADSCs present in the ADSC-implanted animals (B), but absent in the DMEM group animals (C).

labeled ADSCs were observed in the ADSC group showing red fluorescence (Fig. 7B). In contrast, no PKH26 fluorescentlabeled cells were observed in the DMEM group (Fig. 7C).

ADSCs produce supporting factors. Neurotrophic factors and extracellular matrix proteins play an important role in the survival and proliferation of axons and are regarded as axonal growth promoting factors that stimulate axonal regeneration.
Functional benefits derived from cell transplantation include the ability to produce neurotrophic factors. Our experiments showed that cultured ADSCs synthesized and secreted neurothropic factors including NGF, BDNF, NT-3, GDNF, CTNF and LIF (data not shown). To further assess the neurotrophic factor-producing ability of ADSCs after transplantation, total-RNAs were extracted from nerve grafts. Using RT-PCR analysis, we found that at 3 days after surgery, neurotrophic 
A

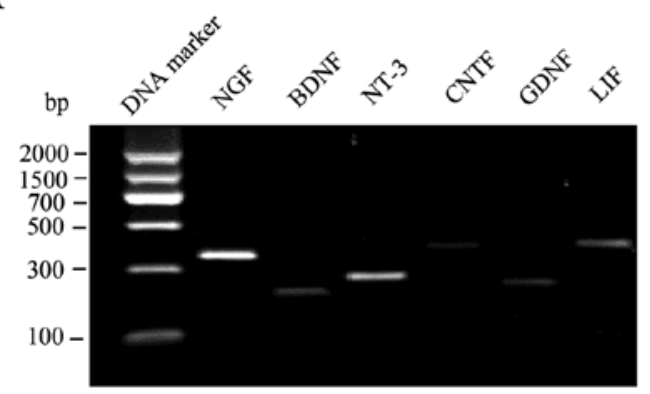

B

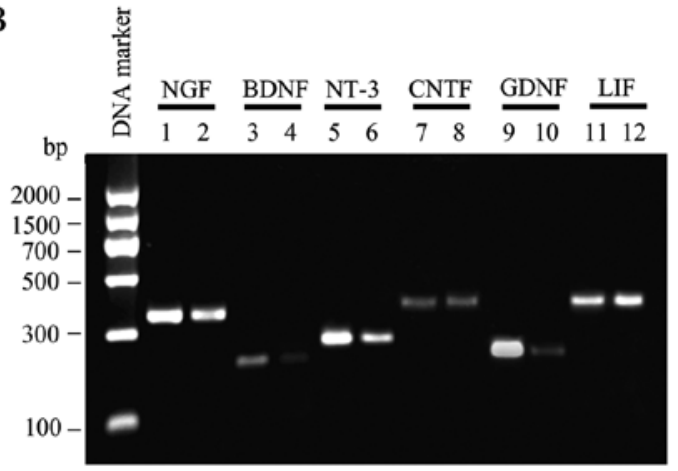

$\mathrm{C}$

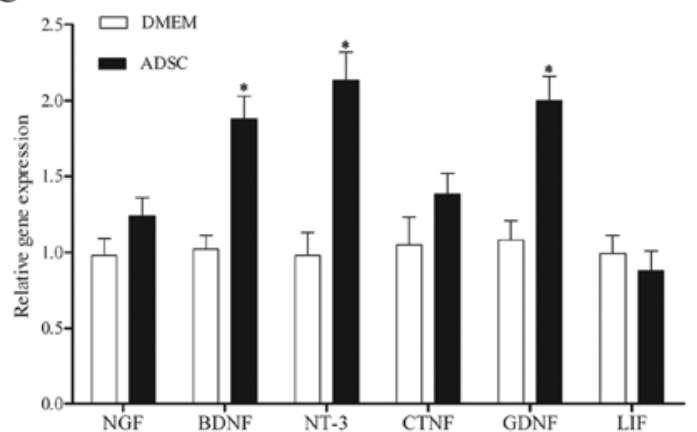

Figure 8. RT-PCR analysis of gene expression in regeneration nerve tissue Total-RNAs were extracted from nerve grafts and subjected to RT-PCR analysis. (A) At 3 days after surgery, neurotrophic factors were expressed in the ADSC-implanted group. (B) At 12 weeks after surgery, neurotrophic factors were expressed in both groups. Lanes 1, 3, 5, 7, 9, 11 represent the ADSC group, and lanes 2, 4, 6, 8, 10, 12 represent the DMEM group. (C) Quantitative analysis revealed that the gene expression of BDNF, NT-3 and GDNF was significantly higher in the ADSC group compared to the DMEM group. ${ }^{*} \mathrm{P}<0.05$

factors including NGF, BDNF, NT-3, GDNF, CTNF and LIF were expressed in the ADSC group (Fig. 8A), but not in the DMEM group (data not shown). At 12 weeks post-surgery, these genes were detected in the nerve grafts from both groups. However, in the ADSC group, BDNF, NT-3 and GDNF were significantly higher expressed in comparison with that in the DMEM group (Fig. 8B and C). These findings demonstrated the high level of neurotrophic factor expression was accompanied by ADSCs implantation in the regenerated nerve tissue.

\section{Discussion}

Neural tissue engineering has recently become the focus of attention in the treatment of peripheral nerve injury. The ideal stem cells for transplantation should be easily obtained, should exhibit rapid proliferation in vitro, low immunogenicity, and should successfully be integrated into the host tissue (25). ADSCs have been proven to possess these characteristics $(12,16)$. In this study, we found that combination of ADSC implantation and ANA graft transplantation can effectively promote the repair of peripheral nerve injury over a $10 \mathrm{~mm}$ gap defect. We showed that ADSC implantation improved the functional recovery of exercise behavior and increased the wet weight ratio of the anterior tibial muscle, indicating that regeneration of nerve fibers in the experimental group enhanced the role of the control and nutrition on the target organs. In the electrophysiological testing, the percentage of activated fibers was higher in the ADSC-implanted group than in the control group as evidenced by the increase of nerve conduction velocity and amplitude. We also showed the increase in myelin thickness after ADSCs implantation, indicated that ADSCs can promote the formation of myelin. Our data suggest that ADSCs can promote the repair of peripheral nerve injury, and combination of ADSCs and ANA transplantation is a new therapeutic method for long distance peripheral nerve defects.

In this study, even though we used the allogeneic cell implantation, we did not observe inflammation or implant rejections. This might be due to the low or absent immunogenicity of ADSC. Also, we found that 12 weeks after transplantation, ADSC transplantation grafts are still alive in the rat body, which further proved that the results of these observations were related to the transplantation of ADSCs, and ADSCs can survive in the body for at least 12 weeks.

Previous studies have shown that neurotrophic factors are essential molecules which can promote early peripheral nerve regeneration $(11,26)$. In our study, cultured ADSCs had the ability to synthesize and release neurotrophic factors such as NGF, BDNF, NT-3, GDNF, CTNF and LIF (data not shown). In comparison with the control group, the implanted ADSCs expressed higher levels of these neurotrophic factors in the early phase of the regenerative process ( 3 weeks after surgery). In the later phase of the regenerative process (12 weeks after surgery), some neurotrophic factors, BDNF, NT-3 and GDNF were significantly higher expressed in the regenerated tissue containing ADSCs implantation compared to the control group. We also found that cultured ADSCs express neural cell adhesion molecules NCAM (data not shown), suggesting that ADSCs may have chemotactic potential in axons extension. In this study, we were unable to study the differentiation of transplanted cells, so it is unclear that these high expressed neurotrophic factors were secreted by the transplanted ADSCs directly or by Schwann cells. However, we demonstrated that the high level of neurotrophic factor expression was accompanied by ADSCs implantation in the regenerated nerve tissue.

In conclusion, we found that ADSCs can survive in the host for long time, and promote the repair of peripheral nerve injury and thus can be used as an ideal cell implantation for tissue engineering. We also provide evidence indicating that the regeneration potential of implanted ADSCs was related to the synthesis and secretion of neurotrophic factors.

\section{Acknowledgements}

This study was supported by grants from the Special Foundation for Science and Technology Research of Shenyang City (Grant 
no. F10-205-1-69), the Foundation for Social Development of Science and Technology Department of Liaoning Province (Grant no. 2010225029), and the Youth Science Foundation of Heilongjiang Province (Grant no. QC2010040).

\section{References}

1. Li Q, Ping $\mathrm{P}$, Jiang $\mathrm{H}$ and Liu K: Nerve conduit filled with GDNF gene-modified Schwann cells enhances regeneration of the peripheral nerve. Microsurgery 26: 116-121, 2006.

2. Mosahebi A, Fuller P, Wiberg M and Terenghi G: Effect of allogeneic Schwann cell transplantation on peripheral nerve regeneration. Exp Neurol 173: 213-223, 2002.

3. Rutkowski GE, Miller CA, Jeftinija S and Mallapragada SK: Synergistic effects of micropatterned biodegradable conduits and Schwann cells on sciatic nerve regeneration. J Neural Eng 1: 151-157, 2004.

4. Pittenger MF, Mackay AM, Beck SC, et al: Multilineage potential of adult human mesenchymal stem cells. Science 284: 143-147, 1999.

5. Cizkova D, Rosocha J, Vanicky I, Jergova S and Cizek M: Transplants of human mesenchymal stem cells improve functional recovery after spinal cord injury in the rat. Cell Mol Neurobiol 26: 1167-1180, 2006.

6. Paul C, Samdani AF, Betz RR, Fischer I and Neuhuber B: Grafting of human bone marrow stromal cells into spinal cord injury: a comparison of delivery methods. Spine (Phila Pa 1976) 34: 328-334, 2009

7. Pal R, Venkataramana NK, Bansal A, et al: Ex vivo-expanded autologous bone marrow-derived mesenchymal stromal cells in human spinal cord injury/paraplegia: a pilot clinical study. Cytotherapy 11: 897-911, 2009.

8. Chen Q, Long Y, Yuan X, et al: Protective effects of bone marrow stromal cell transplantation in injured rodent brain: synthesis of neurotrophic factors. J Neurosci Res 80: 611-619, 2005.

9. Cuevas P, Carceller F, Dujovny M, et al: Peripheral nerve regeneration by bone marrow stromal cells. Neurol Res 24: 634-638, 2002.

10. Cuevas P, Carceller F, Garcia-Gomez I, Yan M and Dujovny M: Bone marrow stromal cell implantation for peripheral nerve repair. Neurol Res 26: 230-232, 2004.

11. Chen CJ, Ou YC, Liao SL, et al: Transplantation of bone marrow stromal cells for peripheral nerve repair. Exp Neurol 204: 443-453, 2007.

12. Zuk PA, Zhu M, Mizuno H, et al: Multilineage cells from human adipose tissue: implications for cell-based therapies. Tissue Eng 7: $211-228,2001$
13. De Ugarte DA, Alfonso Z, Zuk PA, et al: Differential expression of stem cell mobilization-associated molecules on multi-lineage cells from adipose tissue and bone marrow. Immunol Lett 89: 267-270, 2003.

14. De Ugarte DA, Morizono K, Elbarbary A, et al: Comparison of multi-lineage cells from human adipose tissue and bone marrow. Cells Tissues Organs 174: 101-109, 2003.

15. Strem BM, Hicok KC, Zhu M, et al: Multipotential differentiation of adipose tissue-derived stem cells. Keio J Med 54: 132-141, 2005.

16. Kingham PJ, Kalbermatten DF, Mahay D, Armstrong SJ, Wiberg M and Terenghi G: Adipose-derived stem cells differentiate into a Schwann cell phenotype and promote neurite outgrowth in vitro. Exp Neurol 207: 267-274, 2007.

17. Gimble JM, Katz AJ and Bunnell BA: Adipose-derived stem cells for regenerative medicine. Circ Res 100: 1249-1260, 2007.

18. Yoshimura H, Muneta T, Nimura A, Yokoyama A, Koga H and Sekiya I: Comparison of rat mesenchymal stem cells derived from bone marrow, synovium, periosteum, adipose tissue, and muscle. Cell Tissue Res 327: 449-462, 2007.

19. Schaller E, Lassner F, Becker M, Walter GF and Berger A: Regeneration of autologous and allogenic nerve grafts in a rat genetic model: preliminary report. J Reconstr Microsurg 7: 9-12, 1991.

20. Tomaselli KJ, Reichardt LF and Bixby JL: Distinct molecular interactions mediate neuronal process outgrowth on nonneuronal cell surfaces and extracellular matrices. J Cell Biol 103: 2659-2672, 1986

21. Ide C, Osawa T and Tohyama K: Nerve regeneration through allogeneic nerve grafts, with special reference to the role of the Schwann cell basal lamina. Prog Neurobiol 34: 1-38, 1990.

22. Sondell M, Lundborg G and Kanje M: Regeneration of the rat sciatic nerve into allografts made acellular through chemical extraction. Brain Res 795: 44-54, 1998.

23. Bjorntorp P, Karlsson M, Pertoft H, Pettersson P, Sjostrom L and Smith U: Isolation and characterization of cells from rat adipose tissue developing into adipocytes. J Lipid Res 19: 316-324, 1978.

24. Wang D, Liu XL, Zhu JK, et al: Repairing large radial nerve defects by acellular nerve allografts seeded with autologous bone marrow stromal cells in a monkey model. J Neurotrauma 27: 1935-1943, 2010.

25. Barry FP and Murphy JM: Mesenchymal stem cells: clinical applications and biological characterization. Int J Biochem Cell Biol 36: 568-584, 2004.

26. Anton ES, Sandrock AW Jr and Matthew WD: Merosin promotes neurite growth and Schwann cell migration in vitro and nerve regeneration in vivo: evidence using an antibody to merosin, ARM-1. Dev Biol 164: 133-146, 1994. 